CORIGINAL ARTICLE

Volume 15 Issue 12020

DOI: 10.21315/aos2020.15.1.405

ARTICLE INFO

Submitted: $14 / 12 / 2019$

Accepted: 22/06/2020

Online: $15 / 07 / 2020$

\section{Multi-purpose Use of Nasal Stents for Prosthetic Rehabilitation of Facial Defect: A Case Report}

\author{
Waqas Tanveer ${ }^{\mathrm{a}^{*}}$, Pedro Molinero-Mourelle ${ }^{\mathrm{b}}$, Nor Hidayah Reduwanc, \\ Natdhanai Chotprasert ${ }^{\mathrm{d}}$ \\ ${ }^{a}$ Department of Oral and Maxillofacial Surgery, VU University
Medical Center, Amsterdam, The Netherlands \\ ${ }^{b}$ Department of Conservative Dentistry and Orofacial Prosthodontics, \\ Faculty of Dentistry, Complutense University of Madrid, Spain \\ ${ }^{c}$ Centre of Oral and Maxillofacial Diagnostic and Medicine Studies, \\ Faculty of Dentistry, Universiti Teknologi MARA, \\ Sungai Buloh Campus Malaysia, Malaysia \\ ${ }^{d}$ Maxillofacial Prosthetics Service, Faculty of Dentistry, \\ Mahidol University, Bangkok, Thailand
}

*Corresponding author: waqas_tanveer30@hotmail.com

To cite this article: Tanveer W, Molinero-Mourelle P, Reduwan NH, Chotprasert N (2020). Multi-purpose use of nasal stents for prosthetic rehabilitation of facial defect: A case report. Arch Orofac Sci, 15(1): 65-72. https://doi.org/10.21315/aos2020.15.1.405

\begin{abstract}
Basal cell carcinoma is the most common cause of nasal defects. For large lesions with deep tissue extensions a total rhinectomy may be indicated followed by adjunctive therapies, such as radiotherapy or chemotherapy. Subsequent surgical reconstruction of these defects poses a challenge due to the compromised tissue bed and may require rehabilitation with facial prosthesis. In current case report, patient had basal cell carcinoma, which had involved the nasal cartilages, right inferior turbinate, nasolabial fold, upper lip and adjacent vascular and perineural tissues. Basal cell carcinoma was surgically excised, followed by postoperative radiotherapy. The resulted nasal defect was closed by forehead pedicle flap. Nasal stents were inserted postoperatively to prevent stenosis of nostrils. Prosthetic rehabilitation of a complete nasal defect was done by utilising nasal stents to prevent nasal stenosis, improved stability and precision, in placement of prosthesis over flat nasal defect. Skin adhesive was applied on margins to enhance the retention of prosthesis and to blend the margins of prosthesis with adjacent skin.
\end{abstract}

Keywords: Basal cell carcinoma; facial prosthesis; nasal stents; prosthetic rehabilitation

\section{INTRODUCTION}

Partial or complete nasal defects mostly arise due to cancer resection, followed by trauma and congenital anomalies (Nakayama et al., 2011). In particular, the basal cell carcinoma (BCC) is the leading skin cancer of the nose $(32.3 \%)$, which has been attributed to be a result of long-term UV radiation exposure
(Janjua and Qureshi, 2012; Demirseren et al., 2014). Despite it being a slow-growing lesion that renders an early intervention possible, infrequent rare cases depict how the lesion penetrates into the deeper layers of the skin and thus requiring a complete rhinectomy. The consequent nasal reconstruction procedure can be undertaken using various types of flaps, such as bilobed flap, nasolabial 
flap, forehead flap, and septal mucosal flap (Nakayama et al., 2011). However, the compromised tissue bed due to adjunctive radiotherapy therapy can compromise the vascular supply for the reconstruction flap and delay the healing capabilities, resulting in a lower success rate. Therefore, prosthetic rehabilitation serves as an alternative approach to surgical reconstruction in such scenarios. The prostheses are generally retained by, craniofacial implants, adhesives, spectacles, and anatomical undercuts. (Goiato et al., 2012; Negahdari et al., 2014; Shrivastava et al., 2015).

A nasal defect resulting after surgical ablation of tumour can lead to compromised esthetics and functional deficiency. Though, satisfactory aesthetic results have been obtained for partial nasal defect (Malard et al., 2015), but complete nasal defects are preferred over partial nasal defects as they provide freedom in designing retentive attachments as well as prostheses and possibly better marginal adaptation over stable adjacent tissues. Furthermore, following surgical ablation of tumour, postoperative radiation therapy may be required to reduce the chances of reoccurrence. However, this radiation therapy can further pose relative contraindication to craniofacial implants placement for prosthetic rehabilitation, due to compromised blood supply and oxygen level, which adversely affects the survival rate of craniofacial implants (Sinn et al., 2011; Chrcanovic et al., 2016).

The aim of this case report is to present prosthetic rehabilitation of mid-facial defect by utilising silicone nasal stents, which were engaged in the anatomical undercuts of the remaining nostrils. Therefore, nasal stents provided adequate stability and precision of placement of prosthesis on flat nasal defect while maintaining the patency of the nasal airway.

\section{CLINICAL REPORT}

\section{Preprosthetic Surgery and Radiotherapy}

An 80-year-old female patient presented with a complete nasal defect due to the surgical resection of a tumour. Her treatment history revealed that she was diagnosed with BCC (i.e. micro-nodular type, T2N0M0, $>6 \mathrm{~cm}$ ) that extended to involve the nasal cartilages, right inferior turbinate, nasolabial fold, upper lip and adjacent vascular and perineural tissues (Fig. 1). Bilateral partial medial maxillectomy was performed with complete rhinectomy. Forehead pedicle flap was harvested to cover the nasal defect, while an anterior-lateral thigh flap was taken to cover the forehead defect. Additionally, 3400 Gy dose of radiation therapy was given to prevent reoccurrence of tumor. Due to the inadequate size of pedicle flap, postoperative radiotherapy and fibrosis, the upper lip had displaced and constricted towards the nasal defect, resulted in incompetent lips (Fig. 1). Therefore, the patient experienced xerostomia, leakage of fluids through the mouth, and verbal articulation errors. Moreover, persistent oral mucosa exposure to the extra-oral environment inevitably resulted in mouth breathing and hampered proper oral hygiene practices.

The treatment plan was formulated to rehabilitate the mid-facial defect, which involved the fabrication of a silicone facial prosthesis with an upper lip to re-establish the lips competency. The silicone prosthesis was then planned to connect with the nasal stents, which were aimed to maintain nostril patency, improve the stability of prosthesis and to provide precision while placement of prosthesis.

\section{Fabrication of Nasal Prosthesis}

The proposed outline of the facial prosthesis was marked around the nasal and lip defects with an indelible pencil. Meanwhile, the static impression of the nasal and lip defect 
areas was made using soft and hard silicone elastomers (Multisil-Epithetik; Bredent, Poznań, Poland) (Fig. 2). Precautions were taken to prevent the flow of impression materials into the nostrils, specifically by blocking them with soaked gauze. The impression was poured with a Type IV dental stone (Vel-Mix Stone; Kerr Corp, Brea, CA) (Fig. 2). Then, the wax pattern was carved manually and subsequently clinically tested on the patient, while the skin folds and texture were incorporated appropriately in the final wax pattern (Fig. 3). A two-piece mould was fabricated with Type IV dental stone (Vel-Mix Stone; Kerr Corp, Brea, CA) using the lost wax technique (Fig. 4). The Medical Grade silicone VST-50 (Factor II, Lakeside, AZ) was hand mixed according to the manufacturer's recommendation (base to catalyst ratio, 10:1) with internal staining and vulcanised at $100^{\circ} \mathrm{C}$ for 3 hours in a dry heat oven to obtain the facial prosthesis (Fig. 5).
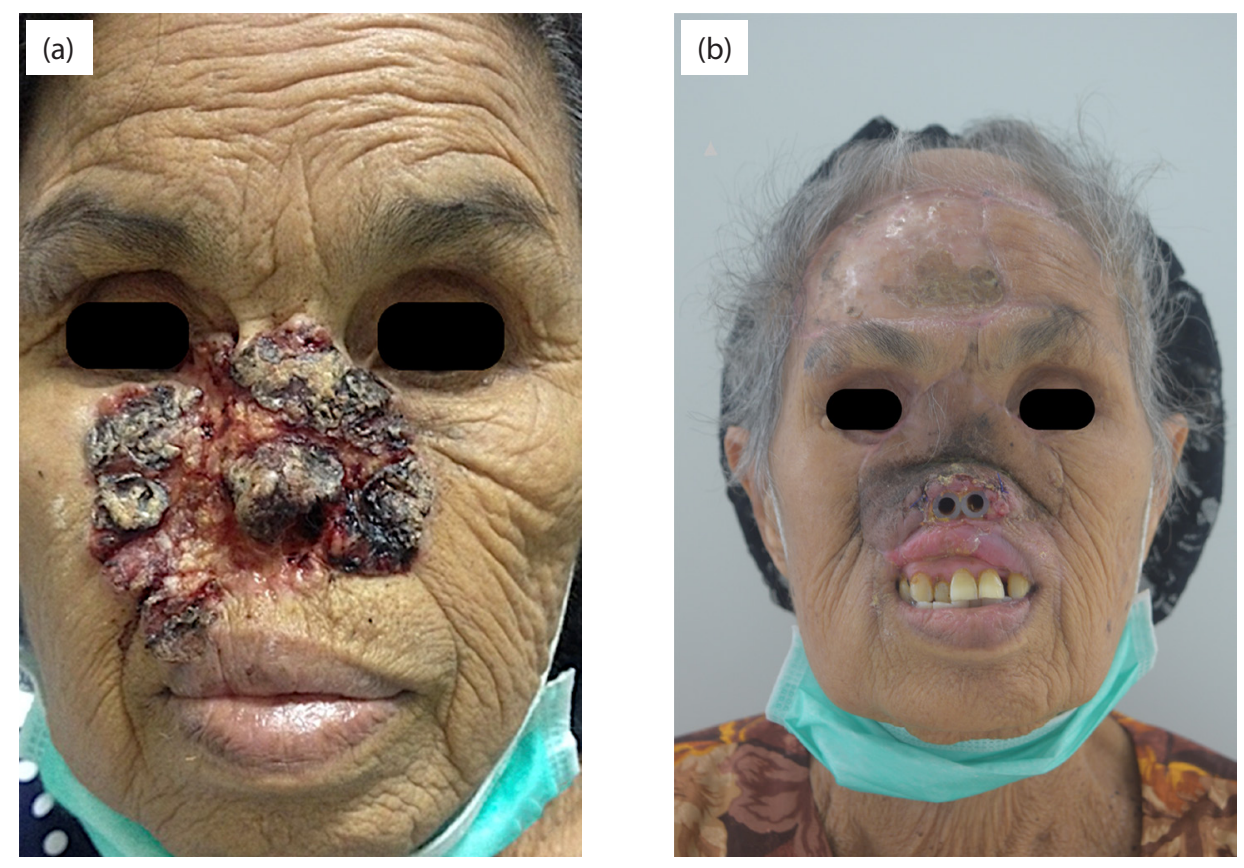

Fig. 1 (a) Initial patient's status with basal cell carcinoma of nose, extending to upper lip. (b) Patient with nasal defect following surgical resection of BCC and radiotherapy. Note the nasal stents in position.
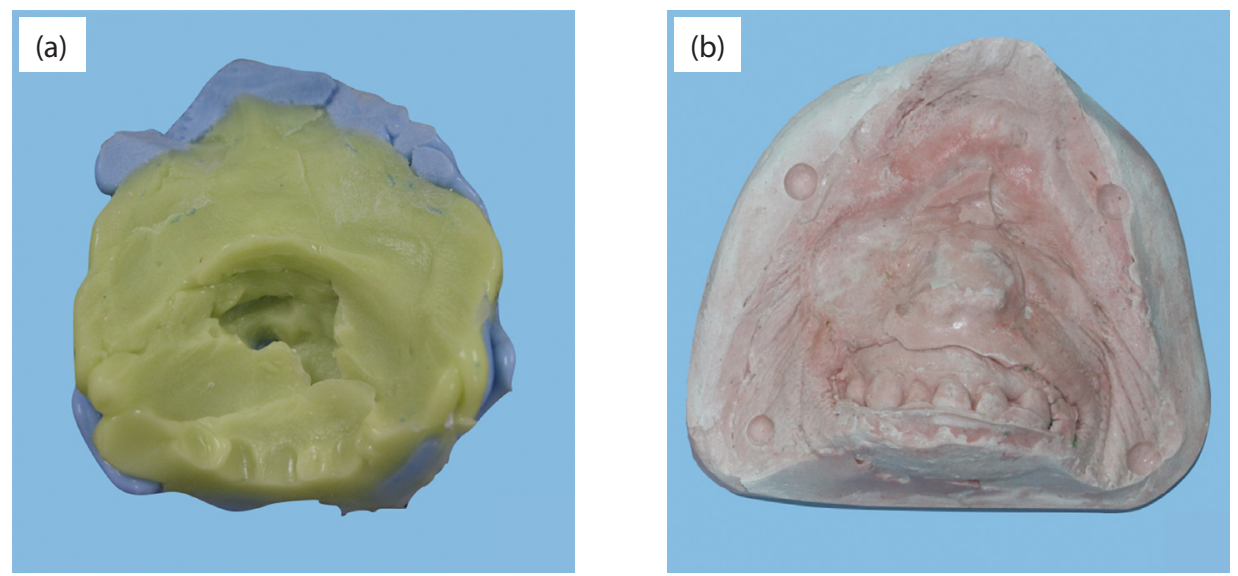

Fig. 2 (a) Impression of nasal defect was made by soft form and hard form silicone impression material.

(b) Impression was poured with Type IV dental stone to obtain working cast. 


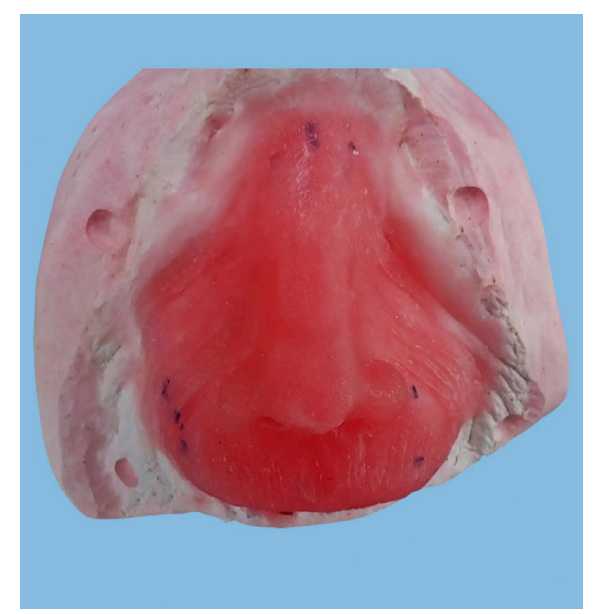

Fig. 3 Final wax pattern after trail procedure. Skin creases and texture incorporated in wax pattern and sealed on working cast.
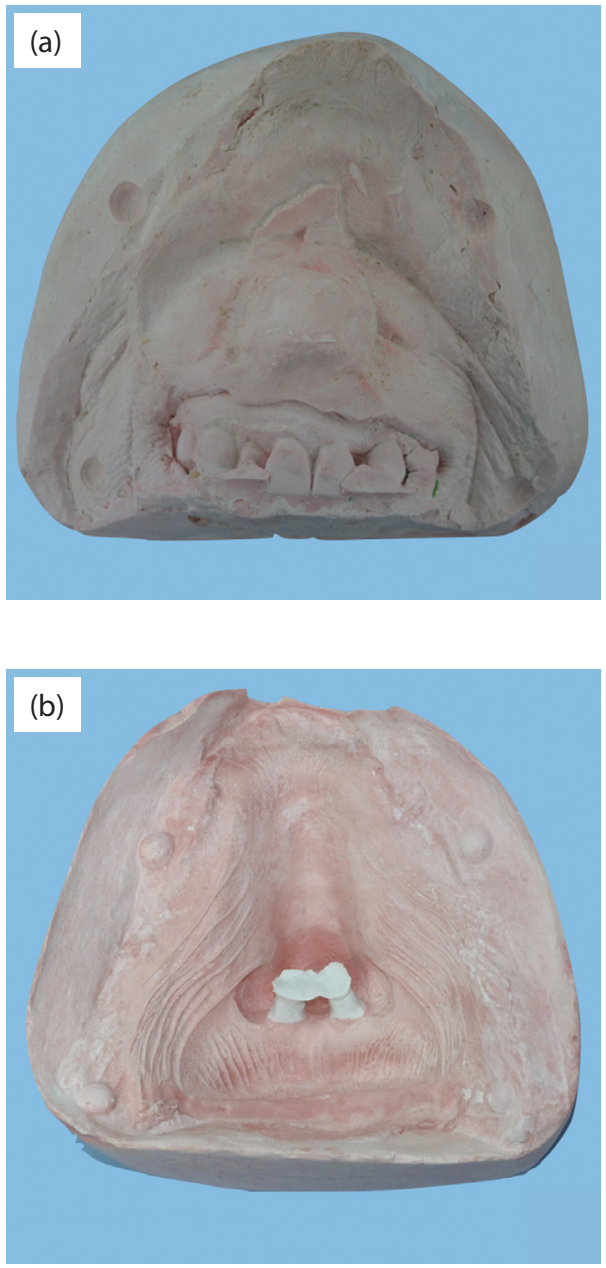

Fig. 4 Mould was fabricated with Type IV dental stone following lost wax technique: (a) Lower part of mould. (b) Upper part of mould.

\section{Process of Nasal Stents Attachment}

Standard medical grade silicone nasopharyngeal airway tubes (Kendall, Mansfield, MA) were obtained and cut into lengths of $3 \mathrm{~cm}$. The size was selected to ensure the overall circumference of each stent was slightly larger than the internal diameter of the nostril. The relationship of the two nasal stents was recorded with a pick-up impression utilising light body VPS impression material (3M ESPE, Seefeld, Germany) (Fig. 6). The impression was poured with Type III dental stone (Sirius; Ultima, Seiches-sur-le-Loir, France) and VPS impression material was replaced with the silicone material LSR-30 (Factor II, Lakeside, AZ) (Fig. 6). Subsequently, the newly fabricated silicone nasal stents assembly was subjected to a clinical trial on the patient upon its insertion into the nostrils (Fig. 7). The nasal stents assembly was then picked up by the vulcanised silicone prosthesis using an A-564 medical adhesive (Factor II, Lakeside, AZ) (Fig. 7). Prior to the pickup procedure, several temporary guidelines were marked on the skin and facial prosthesis to ensure proper orientation. Following trimming of excess adhesive, the new facial prosthesis was inserted after minimal external staining with dry earth pigments (Factor II, Lakeside, AZ) (Fig. 8).

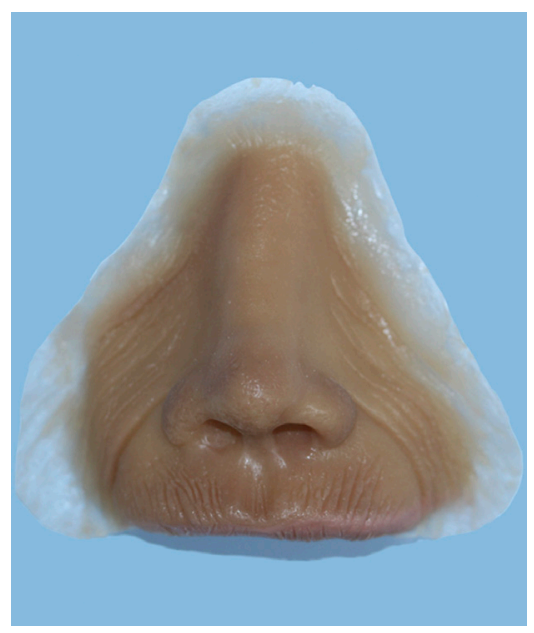

Fig. 5 Cured silicone nasal prosthesis with extended margins. Thin margins were kept intact to blend with adjacent skin. 

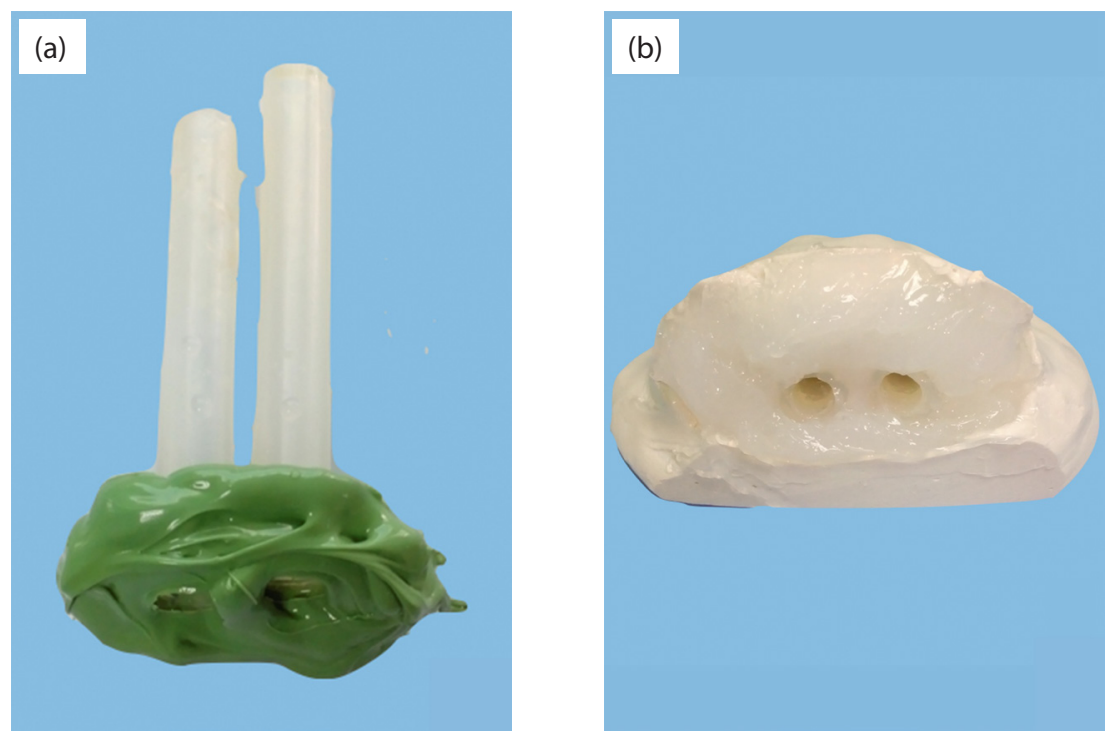

Fig. 6 (a) The position of nasal stents recorded on patient with light body polyvinylsiloxane impression material. (b) After cast fabrication, light body Polyvinylsiloxane impression material was replaced with maxillofacial silicone LSR-30.
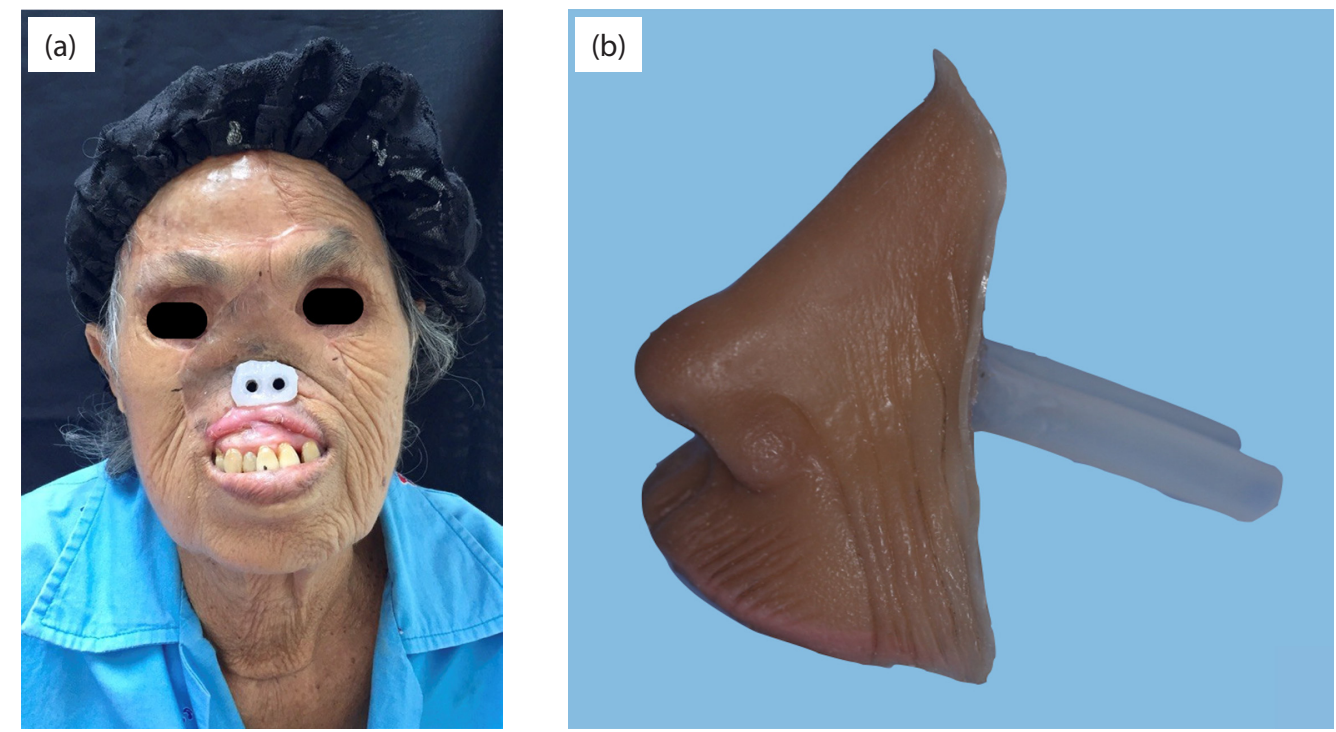

Fig. 7 (a) Nasal stents assembly tried on patient. (b) Nasal stents assembly was picked up with cured silicone nasal prosthesis through medical adhesive.

\section{Patient Instructions and Follow-up}

During resting and sleeping activities, the patient was instructed to remove the facial prosthesis and reinsert the existing postsurgical nasal stents to prevent nasal stenosis. The hygiene instructions were included; cleaning the nasal tubes once daily using warm water with the use of a $10 \mathrm{~mL}$ syringe, gentle cleaning of prosthesis with gauze piece soaked in tap water and cleaning of marginal adhesive around the nasal defect. The patient returned after one, three and six months for follow-up and no signs of inflammation were noted around the nostrils. Additionally, the patient had shown thorough satisfaction with the prosthetic rehabilitation. 


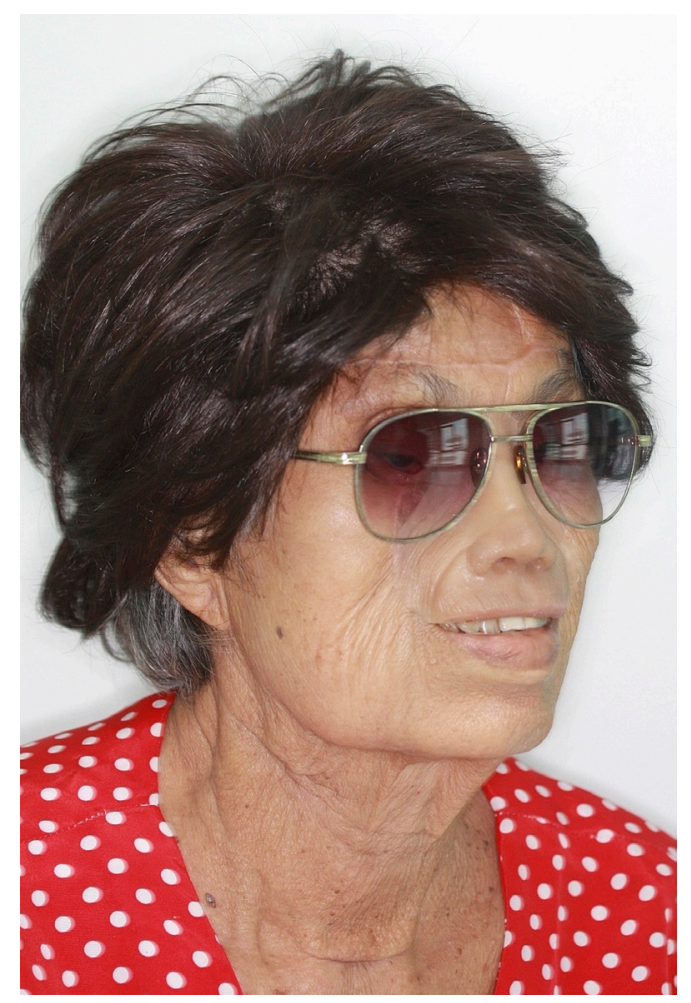

Fig. 8 Definitive outcome after facial prosthesis insertion.

\section{DISCUSSION}

The management of BCC includes, Mohs micrographic surgery, liquid nitrogen, surgical excision, cryosurgery, curettage and electrodessication along with adjuvant radiation therapy and or chemotherapy, depending on the extent, shape, and size of a tumour. Management of these defects can be done by surgical reconstruction or prosthetic rehabilitation. Surgical reconstruction can lead to scaring and contractures therefore it is usually considered only if the size of resulting nasal defect is big enough to compromise the prosthetic outcome. Prosthetic rehabilitation is preferred for nasal defects as prostheses can successfully camouflage the resulting surgical defects. Prosthetic management of nasal defects have been done by conventional methods of fabrication of prosthesis, however since last decade, the trend towards digital planning and fabrication of nasal prostheses has been pushing the limits (Abdullah et al., 2019).
Prosthetic rehabilitation becomes challenging following radiation therapy, chemotherapy, systemic uncontrolled diseases and old age. Lower success and survival rate of craniofacial implants after radiation therapy and chemotherapy have been documented in literature; therefore, placing craniofacial implants in such patients is not always the best option (Malard et al., 2015; Chrcanovic et al., 2016). Furthermore, nostril stenosis is one of the complications that can occur after the surgical resection of a tumour or trauma during healing process, which may lead to scar contractures and decreased patency of nostrils (Egan and Kim, 2005). Thus, alternative means of retention and stability should be sought to improve the aesthetics and functional needs.

In this case, due to postoperative radiotherapy, old age, and financial limitations, implant placement was deemed as an unsuitable option. Using a spectacle for prosthetic retention was considered as another possible option, however, due to the large mid-face prosthesis, retention and stability would be compromised during functional movements. Therefore, silicone nasal stents were utilised to minimise the risk of nostril stenosis and facilitate the retention and stability of the prosthesis. In a previous published report (Goveas et al., 2012), the nasal stent was fabricated with acrylic resin after making an impression which can be a technique sensitive and time-consuming procedure with added disadvantage of rigidity, possibility of bonding failure between acrylic and silicone prosthesis over the period of time, surface roughness within breathing passage leading to retention of nasal secretions and microbial colonisation. In present case, the patient was accustomed to the use of postsurgical nasal stents, therefore, the new prefabricated, flexible silicone nasal stents were taken into consideration while keeping the same dimensions as the existing postsurgical nasal stents and connected them with her newly fabricated mid-facial prosthesis. The silicone nasal stents were preferred over acrylic nasal stents due to the chemical structural affinity 
that existed between the maxillofacial silicone and silicone nasal stents. Therefore, the silicone medical adhesive was thought to provide adequate bond strength between the silicone materials without additional surface treatment and primers. Prior to the medical skin adhesive application, the nasal stents and cured mid-face prosthesis were cleaned with acetone.

Lips incompetency was attributable to upper lip displacement due to the inadequate pedicle forehead flap, postoperative contractures manifested during the healing process, and fibrosis from radiotherapy. Furthermore, constant exposure to the extraoral environment resulted in xerostomia, the difficulty in chewing food, and an inability to maintain meticulous oral hygiene. In this scenario, the silicone nasal prosthesis was extended to fabricate the upper lip and establish lips competency so as to provide a moist intra-oral environment. Therefore, improved oral hygiene in the subsequent follow-up visits was observed after the insertion of the mid-facial prosthesis.

Moreover, the stability of mid-facial prosthesis was achieved by self-guided friction fit silicone nasal stents through the nostrils. Besides, the medical skin adhesive was only applied over the peripheral edges of the prosthesis to ensure smooth blending with the adjacent skin. In subsequent follow up visit, there was no bonding failure noticed between the nasal stents and silicone prosthesis and the skin around the nostrils was normal and showed no signs of inflammation. The addition of an upper lip resulted in lips competency, thus had improved the oral hygiene and masticatory efficiency of the patient. This technique provided the patient with the advantage of precision in prosthesis placement by selfguided nasal stents; therefore, this technique can help patients with problems of manual dexterity while placing prosthesis. The patient can place the prosthesis even without the need of mirror to guide the prosthesis in exact location.

\section{CONCLUSION}

Prefabricated silicone tubes were utilised to ensure nostrils patency, to stabilise the prosthesis, and to provide a guided and established position for easy placement of prosthesis, in the presence of a flat nasal defect. The use of nasal stents limited the application of the medical skin adhesive, thereby rendering the adhesive to be applied only over the edges of the prosthesis to ensure smooth blending with the adjacent skin. Additionally, the inclusion of an upper lip successfully improved the oral hygiene by providing a moist intra-oral environment and establishing the lips competency.

\section{ACKNOWLEDGEMENTS}

The authors want to acknowledge the academic, clinical and laboratory support of Maxillofacial Prosthetic Service, Mahidol University Dental Hospital, especially to Dr Binit Shrestha for his undivided attention and clinical guidance during prosthetic rehabilitation of facial defect. No separate funding was required for this clinical case.

\section{ETHICS STATEMENT}

The case publication is in the scope of the permission to further use medical data signed by every patient at the hospital. The patient signed the general University consent form. This general consent was approved by local ethics committee of University.

\section{CONFLICT OF INTEREST}

No conflict of interest was declared. 


\section{REFERENCES}

Abdullah AM, Mohamad D, Tengku Din TND, Yahya S, Md Akil H, Rajion ZA (2019). Fabrication of nasal prosthesis utilising an affordable 3D Printer. Int $\mathcal{F}$ Adv Manuf Technol, 100: 1907-1912. https://doi.org/ 10.1007/s00170-018-2831-y

Chrcanovic BR, Nilsson J, Thor A (2016). Survival and complications of implants to support craniofacial prosthesis: A systematic review. F Craniomaxillofac Surg, 44(10): 1536-1552. https://doi.org/10 $.1016 /$ j.jcms.2016.07.030

Demirseren DD, Ceran C, Aksam B, Demirseren ME, Metin A (2014). Basal cell carcinoma of the head and neck region: A retrospective analysis of completely excised 331 cases. I Skin Cancer, 2014: 858636. https://doi.org/10.1155/2014/858636

Egan KK, Kim DW (2005). A novel intranasal stent for functional rhinoplasty and nostril stenosis. Laryngoscope, 115(5): 903909. https://doi.org/10.1097/01.MLG $.0000153705 .47361 .1 \mathrm{E}$

Goiato MC, Mancuso DN, Zuccolotti BC, Murakawa AC, Lima DC, Santos DM et al. (2012). Retention and processing methods of nasal prosthesis. F Coll Physicians Surg Pak, 22(11): 716-719.

Goveas R, Puttipisitchet O, Shrestha B, Thaworanunta S, Srithavaj ML (2012). Silicone nasal prosthesis retained by an intranasal stent: A clinical report. f Prosthet Dent, 108(2): 129-132. https:// doi.org/10.1016/S0022-3913(12)60120-6
Janjua OS, Qureshi SM (2012). Basal cell carcinoma of the head and neck region: An analysis of 171 cases. $\mathcal{F}$ Skin Cancer, 2012: 943472. https://doi .org/10.1155/2012/943472

Malard O, Lanhouet J, Michel G, Dreno B, Espitalier F, Rio E (2015). Fullthickness nasal defect: Place of prosthetic reconstruction. Eur Ann Otorhinolaryngol Head Neck Dis, 132(2): 85-89. https://doi .org/10.1016/j.anorl.2014.02.007

Nakayama M, Tabuchi K, Nakamura Y, Hara A (2011). Basal cell carcinoma of the head and neck. I Skin Cancer, 2011: 496910. https://doi.org/10.1155/2011/496910

Negahdari R, Pournasrollah A, Bohlouli S, Sighari Deljavan A (2014). Rehabilitation of a partial nasal defect with facial prosthesis: A case report. $\mathcal{F}$ Dent Res Dent Clin Dent Prospects, 8(4): 256-259. https://doi.org/10.5681/joddd.2014.046

Shrivastava KJ, Shrivastava S, Agarwal S, Bhoyar A (2015). Prosthetic rehabilitation of large mid-facial defect with magnetretained silicone prosthesis. $\mathcal{f}$ Indian Prosthodont Soc, 15(3): 276-280. https://doi .org/10.4103/0972-4052.161571

Sinn DP, Bedrossian E, Vest AK (2011). Craniofacial implant surgery. Oral Maxillofac Surg Clin North Am, 23(2): 321-335, vi-vii. https://doi.org/10.1016/ j.coms.2011.01.005 